\title{
Brain alterations in children/adolescents with ADHD revisited: a neuroimaging meta- analysis of 96 structural and functional studies
}

Fateme Samea ${ }^{1}$ M.Sc., Solmaz Soluki ${ }^{1}$ M.Sc., Vahid Nejati ${ }^{1,2 \neq}$ Ph.D., Mojtaba Zarei ${ }^{3}$ MD., Ph.D., Samuele Cortese ${ }^{4,5,6,7}$ M.D., Ph.D., Simon B. Eickhoff ${ }^{8,9}$ M.D., Masoud Tahmasian ${ }^{3^{\star}}$ M.D., Ph.D., Claudia R. Eickhoff ${ }^{9,10,11}$ M.D.

1 Institute for Cognitive and Brain Sciences, Shahid Beheshti University, Tehran, Iran.

2 Department of Psychology, Shahid Beheshti University, Tehran, Iran.

3 Institute of Medical Science and Technology, Shahid Beheshti University, Tehran, Iran.

4 Center for Innovation in Mental Health, Academic Unit of Psychology, University of Southampton, Southampton, UK.

5 Faculty of Medicine, Clinical and Experimental Sciences (CNS and Psychiatry), University of Southampton, Southampton, UK.

6 Division of Psychiatry and Applied Psychology, School of Medicine, University of Nottingham, Nottingham, UK.

7 Department of Child and Adolescent Psychiatry, NYU Langone Medical Center, New York, USA.

8 Institute for Systems Neuroscience, Medical Faculty, Heinrich-Heine University Düsseldorf, Germany. 9 Institute of Neuroscience and Medicine (INM-1; INM-7), Research Center Jülich, Jülich, Germany. 10 Institute of Clinical Neuroscience and Medical Psychology, Heinrich Heine University Düsseldorf, Düsseldorf, Germany.

11 Department of Psychiatry, Psychotherapy, and Psychosomatics, RWTH Aachen University, Aachen, Germany.

$\neq$ V.N. \& M.T. contributed equally to this study.

* Corresponding author: Masoud Tahmasian M.D., Ph.D., Institute of Medical Science and Technology, Shahid Beheshti University, Daneshjou Boulevard, Velenjak, P.O. Box 1983969411, Tehran, Iran. Telephone: +98-21-29905803; Fax: +98-21-29902650;

Email: m_tahmasian@sbu.ac.ir

Running title: Brain alterations in children/adolescents with ADHD

Count: Title (16), Abstract (168), Text (4263), References (42), Figure (3), Table (1), Supplement file (1).

Key words: ADHD; Activation likelihood estimation; Coordinate-based meta-analysis; fMRI; VBM. 


\section{Abstract}

The findings of neuroimaging studies in children/adolescents with ADHD, and even those of previous meta-analyses, are divergent. Here, Activation Likelihood Estimation meta-analysis, following the current best-practice guidelines, was conducted. We searched multiple databases and traced the references up to June 2018. Then, we extracted the reported coordinates reflecting group comparison between ADHD and healthy subjects from 96 eligible studies, containing 1914 unique participants. The analysis of pooled structural and functional, sub-analyses restricted to modality, and in-/decreased contrast did not yield any significant findings. However, further sub-analyses in the task-fMRI experiments (neutral stimuli only) led to aberrant activity in the left pallidum/putamen and decreased activity (male subjects only) in the left inferior frontal gyrus. The overall findings indicate a lack of regional convergence in children/adolescents with ADHD, which might be due to heterogeneous clinical populations, various experimental design, preprocessing, statistical procedures in individual publications. Our results highlight the need for further high-powered investigations, but may also indicate ADHD pathophysiology might rest in network interactions rather than just regional abnormality. 


\section{Introduction}

Attention-deficit/hyperactivity disorder (ADHD) is a prevalent neurodevelopmental condition characterized by age-inappropriate and impairing inattention and/or impulsiveness-hyperactivity based on Diagnostic and Statistical Manual of Mental Disorders, fifth edition (DSM-5) and International Classification of Diseases (ICD10)(American Psychiatric Association, 2013). ADHD is commonly associated with school/academic, occupational, and social dysfunction (Biederman, 2005; Caye et al., 2016). Furthermore, deficit in several cognitive domains has been demonstrated in individuals with ADHD by neuropsychological studies (SonugaBarke et al., 2010) In addition to neuropsychological, genetics and neurochemical studies investigating the pathophysiology of ADHD (Caye et al., 2016), a large number of neuroimaging studies have been published over the last three decades to elucidate potential structural or functional alterations of ADHD (Hoogman et al., 2017; Konrad and Eickhoff, 2010). However, their findings are often inconsistent or conflicting. Hence, based on these individual studies, the neural correlates of ADHD remain elusive.

Coordinate based meta-analysis (CBMA) of neuroimaging experiments can overcome these issues by providing a synoptic view of findings across studies, hereby consolidating the existing literature (Eickhoff et al., 2012; Turkeltaub et al., 2002). CBMA uses the reported peak coordinates to identify "if" and "where" the convergence between reported coordinates is higher than expected by chance (Eickhoff et al., 2012). Previous meta-analyses on neuroimaging studies in ADHD have focused on structural imaging (Ellison-Wright et al., 2008; Frodl and Skokauskas, 2012; Nakao et al., 2011; Valera et al., 2007) or task-based functional imaging only (Cortese et al., 2012; Dickstein et al., 2006; Hart et al., 2012; Hart et al., 2013; Lei et al., 2015; McCarthy et al., 2014) (Supplemental Table S1). In addition of being outdated, however, they also yielded inconsistent findings. Meta-analyses of structural imaging studies suggested regional gray matter volume reductions in various cortical regions and also the basal ganglia and cerebellum, but showed limited agreement across analyses (Ellison-Wright et al., 2008; Frodl and Skokauskas, 2012; Nakao et al., 2011; Valera et al., 2007). Likewise, meta-analyses of activation studies heterogeneously indicated aberrations in a wide number of regions covering different cerebral lobes and systems as well as the basal ganglia, thalamus and cerebellum (Cortese et al., 2012; Dickstein et al., 2006; Hart et al., 2012; Hart et al., 2013; Lei et al., 2015; McCarthy et al., 2014).

Divergence across previous meta-analyses may in part reflects rather low number of included studies, 
different in-/exclusion criteria, (too) liberal statistical thresholding in search for positive findings (Muller et al., 2018; Tahmasian M, 2018). Moreover, heterogeneity in the examined hypotheses, the assessed clinical populations (e.g. subtype or severity of patients), the applied statistical approaches, and last but not least, different practices in reporting negative findings related to the included individual studies are important factors too (Muller et al., 2018; Tahmasian M, 2018). To address the heterogeneity and statistical power issues of the previous meta-analyses, we conducted a large scale meta-analysis, following recently established consensus guidelines for neuroimaging meta-analyses suggested by the developers of all major software packages (Muller et al., 2018). The current meta-analysis is based on the largest number of original ADHD findings, strict adherence to best-practice protocols and stringent thresholding. Moreover, with regard to heterogeneity issues, we conducted several sub-analyses, clustered by extracted factors representing potential heterogeneity sources (i.e. modality, medication status, gender or behavioral subtype ADHD patients as well as specific cognitive domains and stimuli type of the tasks used in task-fMRI studies). By revisiting the issue of neuroimaging findings in ADHD, we thus aim to settle the ongoing dispute on the presence and localization of structural and/or functional brain alterations in children and adolescents with ADHD.

\section{Methods}

\subsection{Search strategy and selection criteria}

Based on the Preferred Reporting Items for Systematic Reviews and Meta-Analyses(Moher et al.) and current consensus guidelines for neuroimaging meta-analyses (Muller et al., 2018), we searched PubMed, OVID (EMBASE, ERIC and Medline), Web of Knowledge, and Scopus, up to June 1, 2018, for neuroimaging studies on ADHD using the following search terms: (ADHD OR attention-deficit hyperactivity disorder) AND ("functional magnetic resonance imaging" OR fMRI OR "voxel-based morphometry" OR VBM) AND (children OR adolescents). In addition, we identified further papers by reference tracing and consulting review articles. Next, two authors (FS and SS) independently screened all the identified abstracts. Case-reports, letters to editors, reviews, meta-analyses, methodological studies and reports based on < 10 subjects per group were excluded as suggested previously (Muller et al., 2018; Tahmasian et al., 2017; Tahmasian et al., 2016). Furthermore, we excluded all studies using region of interest (ROI) analysis, as the null distribution in CBMA reflects a random spatial association between findings across 
the entire brain. The assumption that each voxel has a priori the same chance for being reported, however, is violated by ROI analyses, creating a sizable bias and inflated significance for the respective regions (Muller et al., 2018; Muller et al., 2017).

Diagnosis of ADHD patients in the included papers had to be based on DSM-IV-TR, or DSM-5, or ICD-10 criteria. The other criteria include the mean age $<18$ year old, no neurological or psychiatric comorbidities (such as depression, anxiety, autism, learning disorder, and epilepsy) or IQ $>70$. We only included the experiments performing a group comparison between patients with ADHD and healthy controls (i.e., no within-group contrasts or contrasts of patients with ADHD versus patients with other disorders). We did not exclude reports on medicated ADHD patients in order to reflect the fact that these represent the bulk of the current literature. However, all included patients were off-medicated during the image acquisition. As suggested (Muller et al., 2018; Muller et al., 2017), studies reporting the effects of pharmacologic or psychological treatment were only included in case the authors reported between-group differences at baseline or main effects of diagnosis.

\subsection{Organization of Coordinates}

Extracted data included bibliographic information, age, gender \& number of subjects, imaging modality, subtype and medication status of patients with ADHD, and the peak coordinates of group comparisons in Montreal Neurological Institute (MNI) (A.C. Evans, 1993) or Talairach (Talairach and Tournoux, 1988) space with the latter being subsequently transformed into MNI space.(Lancaster et al., 2007) Of note, in neuroimaging meta-analyses, "study" refers to a single scientific publication while "experiment" denotes a particular comparison, i.e., a contrast yielding a distinct set of coordinates.

Given that the samples of several studies (partially) overlapped, including such experiments may yield spurious convergence. Thus, we followed the recommended approach to organize data by subjects rather than experiments, avoiding undue influence of a patient cohort (Turkeltaub et al., 2012). Specifically, we combined experiments in the same direction (in-/decreases) for a given set of patients, yielding a maximum of two "experiments" per sample consisting of all extracted coordinates for this set of patients (i.e., one for ADHD > Control contrast, one for Control > ADHD contrast). Of note, the 
experiments were merged regardless of whether separate experiments were reported in one or different papers whenever identity of the subjects could be established (Turkeltaub et al., 2012).

\subsection{Activation likelihood estimation}

We used the revised version of the Activation Likelihood Estimation (ALE) algorithm (Eickhoff et al., 2012) to test significant convergence between activation foci relative to a null-hypothesis of random spatial association between experiments. Firstly, the reported foci were modeled as center peaks of 3D Gaussian probability distributions representing the spatial uncertainty associated with these arising from both sampling effects and methodological differences in data processing and analysis. Importantly, the modeled uncertainty is scaled by the number of subjects in the smaller group to accommodate higher uncertainty of findings from smaller samples. The ensuing per-experiment "modeled activation" maps were then combined into an ALE map by computing their union across experiments (Eickhoff et al., 2012; Turkeltaub et al., 2012). Subsequently, an analytical approach based on non-linear histogram integration was conducted to test against the null hypothesis of random spatial association (Eickhoff et al., 2012; Turkeltaub et al., 2012), followed by cluster-level family-wise error (cFWE) correction at $p<0.05$ (clusterforming threshold of $p<0.001$ on the voxel level) based on Monte-Carlo simulation (Eickhoff et al., 2017; Muller et al., 2018).

\subsection{Performed analyses}

We first performed an ALE analysis across all identified experiments in order to probe for abnormalities independently of direction and neuroimaging modality. Next, we performed three separate sets of analyses assessing increases, decreases or aberrations (pooling in-/decreases) in structural (VBM) and functional (fMRI) imaging, respectively. As recommended, separate analyses were performed only for those combinations of direction and modality yielding at least 17 experiments, given consideration on robustness and power (Eickhoff et al., 2016). Hence, specific analysis of rs-fMRI studies was not possible

due to low number of experiments. Relatedly, we also split the available experiments by medication status, gender or behavioral subtype ADHD patients, noting that the number of experiments for female patients as well as isolated inattentive or hyperactive subtypes was insufficient for analysis. 
Furthermore, we probed for convergence among task-fMRI studies when focusing only on specific cognitive domains and found a sufficient number of experiments (i.e., > 17) (Eickhoff et al., 2016) for inhibitory and attention, but also performed a joint analyses on the remaining cognitive tasks (memory, timing, reasoning). Number of experiments on reward processing tasks, were not sufficient to analyze. Finally, the available studies were also categorized by stimulus type (emotion, reward, neutral), but only field a sufficient number for a sub-analysis of neutral stimuli.

\section{Results}

From a pool of 1586 retrieved publications, 205 potentially eligible studies were identified. Among them, 109 were subsequently excluded due to reasons including ROI analyses, no reported contrast between ADHD and healthy subjects, or reporting of longitudinal effects without baseline. In case the study met all the criteria except reporting the peak coordinates for group comparisons, we contacted the authors to obtain the relevant information. In total, 96 studies were finally eligible for the current meta-analyses (Figure 1, Supplemental Table S2) reporting a total of 130 individual experiments (92 task-fMRI, 32 VBM and 6 rs-fMRI; Supplement) based on 1914 unique subjects. Of these, 62.7\% reported decreases in ADHD patients compared to controls (54 task-fMRI, 25 VBM and 3 rs-fMRI; Supplement).

\subsection{Meta-analyses of neuroimaging findings in ADHD}

Overall, we conducted several separate ALE meta-analyses. When pooling in-/decreases experiments (Figure 2), the results yielded $p=0.129$ (across structural and functional findings), $p=0.452$ (for VBM only), and $p=0.212$ (for fMRI, pooling across task and resting state). Also a restriction to task-fMRI experiments did not provide a significance finding $(p=0.062)$. Similarly, restricting meta-analyses to in/decreases in ADHD, lead to non-significant findings (decreased: all $p=0.195, V B M p=0.341, f M R I p=$ 0.138 , task-fMRI only $p=0.329$; increased: all $p=0.175$; $f M R I p=0.153$, task-fMRI $p=0.668$; Table $1 A)$. Of note, repeating all analyses with an alternative, potentially more liberal statistical threshold (i.e., thresholdfree cluster enhancement, TFCE (Smith and Nichols, 2009)), fully corroborated these results and likewise reveal non-significant convergence (Table $1 \mathrm{~A})$. 


\subsection{Follow-up sub-analyses on task fMRI findings}

Of the 68 task-fMRI studies, 33\% investigated inhibitory control, 29\% attention, $25 \%$ other cognitive domains and 13\% reward processing (Supplement). With respect to stimulus type, $70 \%$ used neutral, $12 \%$ emotional and 17\% rewarding stimuli (Supplement). Again, no significant convergence (Table 1B) was found in any of the more restricted and hence specific sub-analyses for which a sufficient number of experiments was available (inhibitory control $p=0.302$, attention $p=0.847$, all other cognitive domains $p=$ 0.520), even though we noted a trend towards significance when specifically analyzing the convergence of decreased activity for inhibitory control $(p=0.052)$. Repeating all analyses with TFCE threshold, the latter result did pass statistical significance at $p=0.040$ (Table 1B).

We also conducted separate analyses on in-/decreases and their pooled effect for only those experiments using neutral stimuli and found significant aberrant convergence in the left pallidum/putamen (local maximum: -18, 4, -4 in MNI space, 109 voxels, 69.7\% left Pallidum, 12.7\% left Putamen) when looking at the pooled in-/decreased activation experiments in ADHD $(p=0.036)$, but not for either direction individually (decrease $p=0.244$, increase $p=0.110$ (Figure $3 A$, Table $1 C$ ). Finally, we also repeated all analyses including only medication-naïve, male or combined-type ADHD patients, respectively, yielding a single (marginally significant) convergence for fMRI (task-fMRI and rs-fMRI) in male subjects on the left inferior frontal gyrus (IFG) (local maximum: -38, 26, -16 in MNI space, 93 voxels, 83\% in frontal orbital cortex) ( $p=0.049$, Figure 3B, Supplement).

\section{Discussion}

The current study provides the largest and most comprehensive meta-analytic summary of neuroimaging findings in children and adolescents with ADHD across several imaging modalities, following the current best-practice recommendations (Muller et al., 2018). The pooled structural and functional assessment and the additional sub-analyses focusing on homogeneous subsets of experiments (in terms of modality and direction) yielded no significant convergence across the available ADHD literature. Reasons of this heterogeneity may be accounted for several issues including clinical heterogeneity and variations in experimental design and analysis. We discuss further this heterogeneity in the next section. Moreover, we also restricted all the experiments by medication-status, gender, ADHD sub-types, as well as task-fMRI by 
specific cognitive domains and stimulus types, and observed convergence aberrant finding in the taskfMRI experiments (using neutral stimuli) in the left pallidum/putamen and decreased activity (in male subjects only) in the left IFG. Dysfunction of the pallidum/putamen and IFG has been reported in ADHD previously (Castellanos and Proal, 2012; Cortese et al., 2012; Hart et al., 2012; Hart et al., 2013; Konrad and Eickhoff, 2010; Rubia, 2011), which is in line with the fronto-striatal pathway dysfunction model of ADHD. The dopamine-rich ventral putamen receives fibers from the medial orbitofrontal cortex and IFG and it has been shown that their abnormality is linked with hyperactivity, impulsivity, disinhibition, and inattention symptoms of ADHD (Itami and Uno, 2002; Max et al., 2002; Rubia, 2011).

\subsection{Neuroimaging in ADHD: A heterogeneous field}

The heterogeneity in experimental design and procedure across the task-based fMRI experiments, which containing $70 \%$ of our included studies, might be one of the potential sources of inconsistent results. Most available tasks were often aimed at probing various functional impairments in children/adolescents with ADHD (Arnsten and Rubia, 2012). The paradigms of included tasks varied widely (i.e. Go/No-Go, Stroop, Stop signal, timing, reward processing, memory and attention tasks), but each of them applied in less than 10 studies. In addition to the paradigm diversity, there is flexibility in each paradigm regarding to the type of stimuli, presentation of stimuli and type of trials. Due to these inconsistencies, the cognitive operations performed by the subjects when engaging in the respective tasks should vary widely. From the overall inconsistent results of the current study, we conclude that there is limited convergent regional abnormality that was tapped into by the structural and functional experiments that have been used to study ADHD, to date. Additionally, across cognitive domains, there is no convergence in the neurobiological aberrations underlying ADHD. Worthy of note, in the structural imaging studies (i.e. VBM), experimental or design flexibility is absent, but analytical heterogeneity still plays a major role (e.g., various statistical thresholds or spatial transformation of the images). Finally, the included rs-fMRI analytical approaches were likewise vastly different. This methodological multiplicity may have contributed a lot of variance to the current literature beyond what could already be expected given the heterogeneity of the clinical presentation in ADHD. 
Our divergent results on structural and functional experiments support the view that ADHD is a multi-faceted disorder, which affects several functional and behavioral domains. We would hence side with the hypothesis, that ADHD may be primarily reflecting a dysconnectivity disorder, i.e., that the various cognitive and affective affections in ADHD patients may stem from impaired communication and integration between the brain regions/networks rather than from regional abnormalities only (Castellanos and Proal, 2012; Konrad and Eickhoff, 2010). Of note, here we could not test the network alterations due to low number of available rs-fMRI experiment. Also, as recommended, we did not include the ROI-based functional connectivity publications (Muller et al., 2018). In particular, such distributed network pathology would explain both the lack of convergence in either structural or functional imaging findings, as well as the heterogeneous expression of the core and associated symptoms across patients. The latter is particularly important, as ADHD is clinically described as a heterogeneous disorder, which includes impairment on several cognitive and emotional domains (Wahlstedt et al., 2009). These deficits have differential influence on neuropsychological functioning of patients and lead to various clinical presentations (Wahlstedt et al., 2009). Although ADHD is often classified into three different presentations, they have considerable overlap (Wahlstedt et al., 2009). A recent neuroimaging study also found no common brain abnormalities in all ADHD subgroups, suggesting that ADHD is a collection of discrete disorders (Stevens et al., 2018). It is worth mentioning that most of the included studies either recruited patients from different presentations or did not specify the clinical presentation of ADHD that was investigated, even though diagnostic validity of such presentation classification is questionable per se (Bernfeld, 2012). Moreover, most studies retained in the present meta-analysis included participants with high rates of comorbidities, which increases the clinical heterogeneity of the samples (Elia et al., 2008). Thus, even though all patients were labeled as ADHD, they may have presented quite differently, both within each and across samples, potentially featuring different underlying pathologies and hereby further contributing to the lack of convergence using combination of structural and functional experiments. We discuss now key methodological issue form the body of research that we included.

\subsection{Methodological issues}


A main advantage of the ALE meta-analysis approach is integration of a large number of findings to establish consensus on the spatial locations of regional disruptions in neuropsychiatric disorders (Eickhoff et al., 2012; Goodkind et al., 2015; Muller et al., 2017; Tahmasian et al., 2017; Tahmasian et al., 2018; Tahmasian et al., 2016). Thus, ALE allows synthesizing the multitude of single studies in an unbiased fashion and consolidates the available literature, overcoming problems associated with individual neuroimaging experiments (Eickhoff et al., 2012; Muller et al., 2018). Here, we followed the recently developed best-practice protocols of neuroimaging meta-analysis (Muller et al., 2018). In particular, we performed multiple-comparison correction using the cluster-level FWE correction, which should be preferred over the liberal but previously popular false discovery rate (FDR) to provide maximum statistical rigor (Eickhoff et al., 2017; Eickhoff et al., 2016).

In addition, it has been suggested to include at least 17 experiments into an ALE meta-analysis to achieve $80 \%$ power for at least moderate to strong effects (Eickhoff et al., 2016). To explore the potential moderators that might influence on our results, we first identified all eligible experiments and subsequently, categorized them by available variety sources including imaging modality, medication status, gender or behavioral subtype ADHD patients. However, the number of experiments of most of the categories (including rs-fMRI, as well as female patients and isolated inattentive or hyperactive subtypes) were insufficient for a valid analysis. Given that ALE analyses with very few sets of experiments have been shown to be unstable and potentially driven by individual studies, we could not perform the initially planned set of assessments for the effects of most potential moderators. Indeed, exploring moderators is not feasible unless each subgroup yields enough experiments for a separate meta-analysis. Furthermore, by splitting the task-fMRI experiments with specific cognitive domains and also stimulus type of the tasks, the issue was the same, as sufficient number of experiments were not supplied for all subgroups. Accordingly, we could only perform separate meta-analyses for individual subgroups of experiments, categorized by inhibitory, attention, and also joint of the remaining cognitive tasks (memory, timing, and reasoning), as well as neutral stimuli, to probe for convergent functional abnormalities in children and adolescents with ADHD.

Moreover, we carefully excluded studies with ROI analysis to avoid inflating significance for the particular regions (Muller et al., 2018). Importantly, we organized our dataset based on the recommended 
approach, which has been introduced to minimize within-group effects (Turkeltaub et al., 2012) We discuss now our findings in the light of previous ADHD meta-analyses.

\subsection{Divergence of previous mega- and meta-analyses findings}

Previous ADHD meta-analyses focusing on structural imaging (Ellison-Wright et al., 2008; Frodl and Skokauskas, 2012; Nakao et al., 2011; Valera et al., 2007) and task-based fMRI (Cortese et al., 2012; Dickstein et al., 2006; Hart et al., 2012; Hart et al., 2013; Lei et al., 2015; McCarthy et al., 2014) found significant brain abnormalities in variety of brain regions (Supplemental Table S1). Hence, not only individual studies, but also previous meta-analyses in children/adolescents with ADHD have yielded inconsistent findings. This might be due to the fact that those meta-analyses were not following the current best-practice guideline, which is developed recently (Muller et al., 2018). Results of such meta-analyses may be in part driven by small sample size, liberal thresholding and other methodological choices that should be considered non-optimal currently. For example, three meta-analyses on task fMRI (Cortese et al., 2012; Dickstein et al., 2006; Lei et al., 2015) and one on VBM studies (Ellison-Wright et al., 2008) used a version of GingerALE, which was later discovered to have a bug leading to inadequately liberal thresholds that were substantially different from the requested level and did not control for multiple comparisons (Eickhoff et al., 2017). In turn, two fMRI meta-analyses focusing on timing (Hart et al., 2012) and inhibition/attention (Hart et al., 2013), as well as two VBM meta-analyses applied the Signed Differential Mapping (SDM) method (Frodl and Skokauskas, 2012; Nakao et al., 2011), which by design are being more liberal, i.e., to err on the side of false positives rather than negatives. Last but not least, previous meta-analyses assessed substantially smaller samples of experiments, which entail a high likelihood (formally) significant convergence might be often driven by few experiments and dominates the regional effects of those studies (Muller et al., 2018). Given the current growth of the literature, however, we were able to apply more stringent in-/exclusion criteria and still analyze an unprecedentedly large number of individual experiments. Similarly, previous meta-analyses in unipolar depression (Muller et al., 2017) and insomnia disorder (Tahmasian et al., 2018; Tahmasian M, 2018) investigated a large body of literature and followed the best-practice guideline (i.e. rigorous in-/exclusion criteria and statistical threshold), but still observed a lack of convergence across their sub-analyses. The authors suggested that 
small samples of participants, clinical variability, various experimental design, flexibility in preprocessing, and statistical approaches could be the possible reasons of observed heterogeneity in such disorder (Muller et al., 2017; Tahmasian et al., 2018).

Recently, a study comprising 1713 participants with ADHD and 1529 controls from 23 sites applied FreeSurfer and found that volume of the accumbens, amygdala, caudate, hippocampus, putamen, and intracranial volume were smaller in subjects with ADHD compared with controls (9). We did not include this paper, as they focused on particular subcortical ROIs, which could affect the whole brain results. In the present study, we included 28 structural experiments which yielded no significant volume alterations. Of note, the mentioned study has a unified analysis protocol and therefore has less experimental and analytical noise and hence is more likely to find the regional abnormality. However, it is not clear whether the findings are just reflecting their particular regional choices or are robust across studies, which can be tested by neuroimaging meta-analyses.

\subsection{Future directions}

Technical problems and clinical heterogeneity aside, we would argue that one of the major obstacles in neuroimaging research is the strong bias towards publishing positive results, combined with low sample size and high preprocessing and analytical flexibility (garden of forking paths) in (f)MRI analyses. It stands to reason that this combination provides the wrong incentives to analyze data in various ways until significant results emerge, which are often spurious and not replicable or convergent. Coupled with the fact that direct replication studies are still rare, we would thus worry that a large body of the imaging literature contains inflated reports of regional effects which only becomes apparent once well-powered and robust (through their size) meta-analyses as the current are performed. To us, this indicates a need for change at several levels including higher-powered original studies, homogenous clinical populations, preregistration of analysis plans, a better standardization of processing pipelines (also from the level of replicability), large scale open data sharing, and maybe most importantly a cultural shift away from the current bias towards more likely publication of positive findings as well-powered inconsistent findings may be as important to move the entire field forward. 


\section{Conclusion}

To the best of our knowledge, this study is the largest meta-analysis of structural and functional neuroimaging experiments in children/adolescents with ADHD. We found no significant convergent across structural and functional regional alterations in ADHD, which might be attributable to clinical heterogeneity, experimental and analytical flexibility and positive publication bias, but could also point towards a more distributed, network-based pathology lacking a consistent expression at any particular location. Among many different sub-analyses, we only observed the convergence dysfunction for task-fMRI experiments (using neutral stimuli) in the left pallidum/putamen and decreased activity (using male subjects) in the left IFG. This study highlights the need for further exploration assessing regional structural and functional maladaptation, as well as connectivity in parallel to unravel the relation between abnormal regional effects and disturbed integration in ADHD.

\section{Conflict of interest}

The authors declare that the research was conducted in the absence of any commercial or financial relationships that could be construed as a potential conflict of interest.

\section{Acknowledgements}

SBE is supported by the Deutsche Forschungsgemeinschaft DFG (El 816/11-1), the National Institute of Mental Health (R01-MH074457), the Helmholtz Portfolio Theme "Supercomputing and Modeling for the Human Brain" and the European Union's Horizon 2020 Research and Innovation Programme under Grant Agreement No. 785907 (HBP SGA2). 


\section{References}

A.C. Evans, D.L.C., S.R Mills, E.D. Brown, R.L. Kelly, T.M. Peters, 1993. 3D statistical neuroanatomical models from 305 MRI volumes. Nuclear Science Symposium and Medical Imaging Conference, IEEE Conference Record, 1813-1817.

American Psychiatric Association, 2013. Diagnostic and Statistical Manual of Mental Disorders, 5th ed, Washington, DC.

Arnsten, A.F., Rubia, K., 2012. Neurobiological circuits regulating attention, cognitive control, motivation, and emotion: disruptions in neurodevelopmental psychiatric disorders. J Am Acad Child Adolesc Psychiatry 51, 356-367.

Bernfeld, J., 2012. ADHD and factor analysis: are there really three distinct subtypes of ADHD? Appl Neuropsychol Child 1, 100-104.

Biederman, J., 2005. Attention-deficit/hyperactivity disorder: a selective overview. Biol Psychiatry 57, 12151220 .

Castellanos, F.X., Proal, E., 2012. Large-scale brain systems in ADHD: beyond the prefrontal-striatal model. Trends Cogn Sci 16, 17-26.

Caye, A., Rocha, T.B., Anselmi, L., Murray, J., Menezes, A.M., Barros, F.C., Goncalves, H., Wehrmeister, F., Jensen, C.M., Steinhausen, H.C., Swanson, J.M., Kieling, C., Rohde, L.A., 2016. Attention-Deficit/Hyperactivity Disorder Trajectories From Childhood to Young Adulthood: Evidence From a Birth Cohort Supporting a Late-Onset Syndrome. JAMA Psychiatry 73, 705-712.

Cortese, S., Kelly, C., Chabernaud, C., Proal, E., Di Martino, A., Milham, M.P., Castellanos, F.X., 2012. Toward systems neuroscience of ADHD: a meta-analysis of 55 fMRI studies. Am J Psychiatry 169, 1038-1055.

Dickstein, S.G., Bannon, K., Castellanos, F.X., Milham, M.P., 2006. The neural correlates of attention deficit hyperactivity disorder: an ALE meta-analysis. J Child Psychol Psychiatry 47, 1051-1062.

Eickhoff, S.B., Bzdok, D., Laird, A.R., Kurth, F., Fox, P.T., 2012. Activation likelihood estimation meta-analysis revisited. Neuroimage 59, 2349-2361.

Eickhoff, S.B., Laird, A.R., Fox, P.M., Lancaster, J.L., Fox, P.T., 2017. Implementation errors in the GingerALE Software: Description and recommendations. Hum Brain Mapp 38, 7-11.

Eickhoff, S.B., Nichols, T.E., Laird, A.R., Hoffstaedter, F., Amunts, K., Fox, P.T., Bzdok, D., Eickhoff, C.R., 2016. Behavior, sensitivity, and power of activation likelihood estimation characterized by massive empirical simulation. Neuroimage 137, 70-85.

Elia, J., Ambrosini, P., Berrettini, W., 2008. ADHD characteristics: I. Concurrent co-morbidity patterns in children \& adolescents. Child Adolesc Psychiatry Ment Health 2, 15.

Ellison-Wright, I., Ellison-Wright, Z., Bullmore, E., 2008. Structural brain change in Attention Deficit Hyperactivity Disorder identified by meta-analysis. BMC psychiatry 8, 51.

Frodl, T., Skokauskas, N., 2012. Meta-analysis of structural MRI studies in children and adults with attention deficit hyperactivity disorder indicates treatment effects. Acta psychiatrica Scandinavica 125, 114-126. 
Goodkind, M., Eickhoff, S.B., Oathes, D.J., Jiang, Y., Chang, A., Jones-Hagata, L.B., Ortega, B.N., Zaiko, Y.V., Roach, E.L., Korgaonkar, M.S., Grieve, S.M., Galatzer-Levy, I., Fox, P.T., Etkin, A., 2015. Identification of a common neurobiological substrate for mental illness. JAMA Psychiatry 72, 305-315.

Hart, H., Radua, J., Mataix-Cols, D., Rubia, K., 2012. Meta-analysis of fMRI studies of timing in attention-deficit hyperactivity disorder (ADHD). Neurosci Biobehav Rev 36, 2248-2256.

Hart, H., Radua, J., Nakao, T., Mataix-Cols, D., Rubia, K., 2013. Meta-analysis of functional magnetic resonance imaging studies of inhibition and attention in attention-deficit/hyperactivity disorder: exploring task-specific, stimulant medication, and age effects. JAMA Psychiatry 70, 185-198.

Hoogman, M., Bralten, J., Hibar, D.P., Mennes, M., Zwiers, M.P., Schweren, L.S.J., van Hulzen, K.J.E., Medland, S.E., Shumskaya, E., Jahanshad, N., Zeeuw, P., Szekely, E., Sudre, G., Wolfers, T., Onnink, A.M.H., Dammers, J.T., Mostert, J.C., Vives-Gilabert, Y., Kohls, G., Oberwelland, E., Seitz, J., Schulte-Ruther, M., Ambrosino, S., Doyle, A.E., Hovik, M.F., Dramsdahl, M., Tamm, L., van Erp, T.G.M., Dale, A., Schork, A., Conzelmann, A., Zierhut, K., Baur, R., McCarthy, H., Yoncheva, Y.N., Cubillo, A., Chantiluke, K., Mehta, M.A., Paloyelis, Y., Hohmann, S., Baumeister, S., Bramati, I., Mattos, P., Tovar-Moll, F., Douglas, P., Banaschewski, T., Brandeis, D., Kuntsi, J., Asherson, P., Rubia, K., Kelly, C., Martino, A.D., Milham, M.P., Castellanos, F.X., Frodl, T., Zentis, M., Lesch, K.P., Reif, A., Pauli, P., Jernigan, T.L., Haavik, J., Plessen, K.J., Lundervold, A.J., Hugdahl, K., Seidman, L.J., Biederman, J., Rommelse, N., Heslenfeld, D.J., Hartman, C.A., Hoekstra, P.J., Oosterlaan, J., Polier, G.V., Konrad, K., Vilarroya, O., Ramos-Quiroga, J.A., Soliva, J.C., Durston, S., Buitelaar, J.K., Faraone, S.V., Shaw, P., Thompson, P.M., Franke, B., 2017. Subcortical brain volume differences in participants with attention deficit hyperactivity disorder in children and adults: a cross-sectional mega-analysis. Lancet Psychiatry 4, 310-319.

Itami, S., Uno, H., 2002. Orbitofrontal cortex dysfunction in attention-deficit hyperactivity disorder revealed by reversal and extinction tasks. Neuroreport 13, 2453-2457.

Konrad, K., Eickhoff, S.B., 2010. Is the ADHD brain wired differently? A review on structural and functional connectivity in attention deficit hyperactivity disorder. Hum Brain Mapp 31, 904-916.

Lancaster, J.L., Tordesillas-Gutierrez, D., Martinez, M., Salinas, F., Evans, A., ZilleS, K., Mazziotta, J.C., Fox, P.T., 2007. Bias between MNI and talairach coordinates analyzed using the ICBM-152 brain template. Human Brain Mapping 28, 1194-1205.

Lei, D., Du, M., Wu, M., Chen, T., Huang, X., Du, X., Bi, F., Kemp, G.J., Gong, Q., 2015. Functional MRI reveals different response inhibition between adults and children with ADHD. Neuropsychology 29, 874-881.

Max, J.E., Fox, P.T., Lancaster, J.L., Kochunov, P., Mathews, K., Manes, F.F., Robertson, B.A., Arndt, S., Robin, D.A., Lansing, A.E., 2002. Putamen lesions and the development of attention-deficit/hyperactivity symptomatology. J Am Acad Child Adolesc Psychiatry 41, 563-571.

McCarthy, H., Skokauskas, N., Frodl, T., 2014. Identifying a consistent pattern of neural function in attention deficit hyperactivity disorder: a meta-analysis. Psychol Med 44, 869-880.

Moher, D., Liberati, A., Tetzlaff, J., Altman, D.G., Group, P., 2009. Preferred reporting items for systematic reviews and meta-analyses: the PRISMA statement. PLoS Med 6, e1000097.

Muller, V.I., Cieslik, E.C., Laird, A.R., Fox, P.T., Radua, J., Mataix-Cols, D., Tench, C.R., Yarkoni, T., Nichols, T.E., Turkeltaub, P.E., Wager, T.D., Eickhoff, S.B., 2018. Ten simple rules for neuroimaging meta-analysis. Neurosci Biobehav Rev 84, 151-161.

Muller, V.I., Cieslik, E.C., Serbanescu, I., Laird, A.R., Fox, P.T., Eickhoff, S.B., 2017. Altered Brain Activity in Unipolar Depression Revisited: Meta-analyses of Neuroimaging Studies. JAMA Psychiatry 74, 47-55. 
Nakao, T., Radua, J., Rubia, K., Mataix-Cols, D., 2011. Gray matter volume abnormalities in ADHD: voxel-based meta-analysis exploring the effects of age and stimulant medication. Am J Psychiatry 168, 1154-1163.

Rubia, K., 2011. "Cool" inferior frontostriatal dysfunction in attention-deficit/hyperactivity disorder versus "hot" ventromedial orbitofrontal-limbic dysfunction in conduct disorder: a review. Biol Psychiatry 69, e69-87.

Smith, S.M., Nichols, T.E., 2009. Threshold-free cluster enhancement: Addressing problems of smoothing, threshold dependence and localisation in cluster inference. Neuroimage 44, 83-98.

Sonuga-Barke, E., Bitsakou, P., Thompson, M., 2010. Beyond the dual pathway model: evidence for the dissociation of timing, inhibitory, and delay-related impairments in attention-deficit/hyperactivity disorder. J Am Acad Child Adolesc Psychiatry 49, 345-355.

Stevens, M.C., Pearlson, G.D., Calhoun, V.D., Bessette, K.L., 2018. Functional Neuroimaging Evidence for Distinct Neurobiological Pathways in Attention-Deficit/Hyperactivity Disorder. Biol Psychiatry Cogn Neurosci Neuroimaging 3, 675-685.

Tahmasian, M., Eickhoff, S.B., Giehl, K., Schwartz, F., Herz, D.M., Drzezga, A., van Eimeren, T., Laird, A.R., Fox, P.T., Khazaie, H., Zarei, M., Eggers, C., Eickhoff, C.R., 2017. Resting-state functional reorganization in Parkinson's disease: An activation likelihood estimation meta-analysis. Cortex 92, 119-138.

Tahmasian M, N.K., Samea F, Zarei M, Spiegelhalder K, Eickhoff SB, Van Someren E, Khazaie H, Eickhoff CR., 2018. Reply to Hua Liu, HaiCun Shi and PingLei Pan: Coordinate based meta-analyses in a medium sized literature: considerations, limitations and road ahead. Sleep Medicine Reviews.

Tahmasian, M., Noori, K., Samea, F., Zarei, M., Spiegelhalder, K., Eickhoff, S.B., van Someren, E., Khazaie, H., Eickhoff, C.R., 2018. A Lack of consistent brain alterations in insomnia disorder: an activation likelihood estimation meta-analysis. Sleep Medicine Reviews.

Tahmasian, M., Rosenzweig, I., Eickhoff, S.B., Sepehry, A.A., Laird, A.R., Fox, P.T., Morrell, M.J., Khazaie, H., Eickhoff, C.R., 2016. Structural and functional neural adaptations in obstructive sleep apnea: an activation likelihood estimation meta-analysis. Neurosci Biobehav Rev.

Talairach, J., Tournoux, P., 1988. Co-planar stereotaxic atlas of the human brain : 3-dimensional proportional system : an approach to cerebral imaging. Georg Thieme, Stuttgart ; New York.

Turkeltaub, P.E., Eden, G.F., Jones, K.M., Zeffiro, T.A., 2002. Meta-analysis of the functional neuroanatomy of single-word reading: method and validation. Neuroimage 16, 765-780.

Turkeltaub, P.E., Eickhoff, S.B., Laird, A.R., Fox, M., Wiener, M., Fox, P., 2012. Minimizing within-experiment and within-group effects in Activation Likelihood Estimation meta-analyses. Hum Brain Mapp 33, 1-13.

Valera, E.M., Faraone, S.V., Murray, K.E., Seidman, L.J., 2007. Meta-analysis of structural imaging findings in attention-deficit/hyperactivity disorder. Biol Psychiatry 61, 1361-1369.

Wahlstedt, C., Thorell, L.B., Bohlin, G., 2009. Heterogeneity in ADHD: neuropsychological pathways, comorbidity and symptom domains. J Abnorm Child Psychol 37, 551-564. 


\section{Figures' legends}

Figure 1. Study selection strategy flow chart. ROI: region of Interest, task-fMRI: task-based functional magnetic resonance imaging, rs-fMRI: resting-state functional magnetic resonance imaging, VBM: voxelbased morphometry, ADHD: attention-deficit/hyperactivity disorder.

Figure 2. Distribution of the included coordinates in the current study. Included coordinates reflecting structural/functional alterations in children/adolescents with ADHD compared to healthy subjects. Rs-fMRI: resting-state functional magnetic resonance imaging; task-fMRI: task-based functional magnetic resonance imaging; VBM: voxel-based morphometry.

Figure 3. Convergent findings of sub-analyses. A. Aberrant activity (green) in the left pallidum/putamen $(-18,4,-4 \mathrm{MNI}, 109$ voxels, $\mathrm{p}=0.036)$, based on task-based fMRI experiments (using neutral stimuli only); B. Decreased activity (blue) in the left inferior frontal gyrus $(-38,26,-16 \mathrm{MNI}, 93$ voxels, $p=0.049$ based on fMRI (task-fMRI \& rs-fMRI) experiments (using male patients only). 
Table 1: Results of conducted meta-analyses in children/adolescent with ADHD compared to healthy subjects

\begin{tabular}{|c|c|c|c|c|c|}
\hline \multicolumn{6}{|c|}{ A. Modality } \\
\hline & \multirow{2}{*}{$\begin{array}{c}\text { Number of } \\
\text { Experiments }\end{array}$} & \multirow{2}{*}{ Modalities } & \multirow{2}{*}{ Contrasts } & \multicolumn{2}{|c|}{ P-value } \\
\hline & & & & TFCE & cFWE \\
\hline 1 & 130 & VBM, task-fMRI, rs-fMRI & - & 0.170 & 0.129 \\
\hline 2 & 81 & VBM, task-fMRI, rs-fMRI & Control > ADHD & 0.087 & 0.195 \\
\hline 3 & 48 & VBM, task-fMRI, rs-fMRI & ADHD > Control & $0 \cdot 104$ & 0.175 \\
\hline 4 & 98 & $\begin{array}{c}\mathrm{fMRI} \\
\text { (combination of task- and rs-fMRI) }\end{array}$ & - & $0 \cdot 151$ & $0 \cdot 212$ \\
\hline 5 & 57 & $\begin{array}{c}\mathrm{fMRI} \\
\text { (combination of task- and rs-fMRI) }\end{array}$ & Control > ADHD & 0.061 & $0 \cdot 138$ \\
\hline 6 & 1 & $\begin{array}{c}\mathrm{fMRI} \\
\text { (combination of task- and rs-fMRI) }\end{array}$ & ADHD > Control & 0.063 & $0 \cdot 153$ \\
\hline 7 & 92 & task-fMRI & - & 0.064 & 0.062 \\
\hline 8 & 54 & task-fMRI & Control > ADHD & 0.103 & 0.329 \\
\hline 9 & 38 & task-fMRI & ADHD > Control & 0.248 & 0.668 \\
\hline 10 & 32 & VBM & - & 0.817 & 0.452 \\
\hline 11 & 25 & VBM & Control > ADHD & 0.630 & 0.341 \\
\hline \multicolumn{6}{|c|}{ B. Cognitive domain } \\
\hline & \multirow{2}{*}{$\begin{array}{c}\text { Number of } \\
\text { Experiments }\end{array}$} & \multirow{2}{*}{ Domain } & \multirow{2}{*}{ Contrasts } & \multicolumn{2}{|c|}{ P-value } \\
\hline & & & & TFCE & cFWE \\
\hline 12 & 31 & Inhibitory control & - & 0.405 & 0.302 \\
\hline 13 & 18 & Inhibitory control & Control > ADHD & 0.040 & 0.052 \\
\hline 15 & 27 & Attention & - & 0.447 & 0.847 \\
\hline 18 & 23 & Other cognitive domains & - & 0.440 & 0.520 \\
\hline \multicolumn{6}{|c|}{ C. Stimulus type } \\
\hline & \multirow{2}{*}{$\begin{array}{c}\text { Number of } \\
\text { Experiments }\end{array}$} & \multirow{2}{*}{ Stimulus Type } & \multirow{2}{*}{ Contrasts } & \multicolumn{2}{|c|}{ P-value } \\
\hline & & & & TFCE & CFWE \\
\hline 21 & 65 & Neutral & - & 0.079 & 0.036 \\
\hline 22 & 41 & Neutral & Control > ADHD & 0.048 & 0.244 \\
\hline 23 & 24 & Neutral & ADHD > Control & 0.104 & 0.110 \\
\hline
\end{tabular}

rs-fMRI: resting-state functional magnetic resonance imaging; task-fMRI: task-based functional magnetic resonance imaging; VBM: voxel-based morphometry; TFCE: threshold-free cluster enhancement; cFWE: cluster-level family-wise error. 